Kathrine Sørensen Ravn Jørgensen*

\title{
Le présent historique et ses fonctions textuelles
}

\begin{abstract}
According to some grammarians the use of the historical present tense is a technique for enhancing the dramatic effect of a story by making addressees feel as if they were present at the time of the experience, witnessing events as they occurred. Others have suggested that the historical present represents events as if they were occurring before the speaker's eyes. My own position on the matter, though, is that the necessity to postulate a "metaphorical shift", an "as if", is due to the interpretation of the basic meaning of the present as being contemporality with the speaker's present and the identification of a speaker (narrator) with an "intradiegetic" participant in the story (character). In support of this position the paper argues that the temporal referential of the present tense is the speech referential, organised by and around the speaker/character. The speech process is an incomplete process with a validation interval closed to the left and open to the right. This property motivates use of the present as a grammatical vehicle for re-presentation.
\end{abstract}

\section{Indledning}

Toujours mentionné en seconde place dans les grammaires, le présent historique est classé parmi les cas particuliers - M. Wilmet parle même "des emplois impurs" du présent -. En effet, le paragraphe traitant du présent de l'indicatif commence toujours par une définition assimilant le présent, forme verbale, au présent vécu, c'est-à-dire à l'actuel du locuteur, et fait de cette coïncidence la "véritable" valeur de la forme du présent. Par conséquent, le présent historique qui déroge à cette coïncidence ne peut être considéré que comme une distorsion à effet stylistique. P. Imbs le décrit comme un "fait de syntaxe expressive", H. Weinrich parle de "métaphore temporelle", E. Benveniste parle d'un "simple artifice de style" et R. Martin considère que ce temps "confère au récit une certaine vivacité".

* Kathrine Sørensen Ravn Jørgensen

Institut for fransk

Handelsh фjskolen i Kфbenhavn

Dalgas Have 15

DK-2000 Frederiksberg 
Trouvant avec raison l'approche temporelle du temps insatisfaisante, M. Herslund propose une approche d'allure modale selon laquelle ce qui importe, ce n'est pas le locuteur, en tant que centre temporel déictique, mais son point de vue sur la situation décrite. Dans cette perspective, la fonction textuelle du présent historique est de commenter le monde. Or, nous allons démontrer que le locuteur, par l'emploi du présent historique, ne commente pas le monde mais qu'il le représente en direct, qu'il nous le montre.

Tout en nous inspirant des analyses de Herslund, nous allons proposer une approche aspectuelle du présent historique, qui mettra en évidence sa valeur d'inaccompli. D'après cette approche, l'impression du vécu en direct est due à la saisie ouverte du procès qui évoque l'image d'un processus en cours, comparable au processus de l'énonciation qui le produit.

A l'approche aspectuelle, nous ajouterons l'approche du repérage temporel de A.Culioli et de M. Vuillaume. Selon Culioli, le présent historique n'est pas repéré par rapport au moment de parole mais par rapport à un repère-origine fictif, c'est-à-dire que le moment du locuteur n'a pas de valeur propre et que c'est le moment du lecteur qui actualise le moment du locuteur et lui donne une valeur. Cela explique la question du "transport" du locuteur ou de l'événement et l'indécision des lecteurs devant ce type de formes : ils les interprètent à la fois comme des événements dissociés du présent et comme la réactualisation, la "résurrection" de faits révolus. Le repère-origine fictif correspond au repérage de la fiction secondaire de Vuillaume.

A travers l'étude de textes de divers types, nous appliquerons les différentes approches, et dans notre étude des récurrences du présent historique, il apparaîtra que sa valeur temporelle n'est qu'un des effets de sens résultant de la combinatoire des valeurs de modalité, d'aspect et de repérage.

\section{Les approches traditionnelles du temps comme catégo- rie déictique}

Aristote a énoncé la théorie des trois époques, des trois tiroirs temporels dans lesquels prend obligatoirement place selon lui, toute forme verbale. Il chante se situe dans un "maintenant" ; un sens "actuel" lui est inhérent, comme un sens "passé" à il chantait, et un sens "futur" à $i l$ 
chantera. Cette tripartition temporelle a été développée jusqu'au XXe siècle par les linguistes qui conçoivent le temps comme une catégorie déictique. Ainsi trouve-t-on chez P. Imbs (1960) la traditionnelle tripartition du temps en passé, présent et futur. Imbs conçoit son système temporel comme un réseau de relations, qui prend son origine dans le présent ; les autres temps se définissent par une relation d'antériorité ou de postériorité par rapport à ce présent ou par rapport au passé et au futur. Son expression "origine des temps" pour le présent semble suggérer le caractère déictique de son système, même si cela reste un peu vague. Dans les systèmes proposés par des linguistes français, tels que E. Benveniste et R. Martin ou ceux proposés par des linguistes anglosaxons, tels que J. Lyons et B. Comrie, le moment de la parole joue explicitement un rôle essentiel dans la catégorisation du temps. Chez ces auteurs, le temps, en tant que catégorie déictique, relie le discours au moment de la parole et en structure le contenu en situations qui sont reliées les unes aux autres et au moment de la parole. Selon cette conception, un verbe au présent, au passé et au futur indiquent respectivement la simultanéité, l'antériorité et la postériorité de la situation décrite par rapport au moment de la parole.

Même si la conception du temps comme une catégorie déictique a pour elle l'évidence du bon sens, chacun de nous ressentant ainsi le temps puisque nous vivons, pensons et parlons dans un maintenant, un avant, et un après, cette intuition psychologique n'a pas forcément une valeur grammaticale. Si le signifiant "présent" avait pour signifié "actuel", comment pourrait-on expliquer l'usage si fréquent du "présent pour le passé" et "du présent pour le futur". Il en va de même des imparfaits qui ne renvoient pas au passé : les exemples d'imparfait à valeur contrefactuelle si j'étais... ou imparfait de politesse je voulais vous demander... Et le futur renvoyant au présent je vous avouerai que.... C'est pourquoi les formes de présent, de passé et de futur qui n'ont pas leur valeur temporelle habituelle sont souvent classées dans les grammaires parmi les cas particuliers.

\section{L'approche modo-temporelle}

Il faut donc admettre que la conception temporelle du temps ne peut pas expliquer tous les emplois des formes temporelles. Cela conduit M. Herslund à proposer une "conception [qui] ne sera pas fondée sur l'opposition entre le 'passé', le 'présent' et le 'futur'[...]. Une distinction 
qui semble plus pertinente est celle entre 'l'actuel' et le 'non-actuel', c'est-à-dire entre ce qui est le cas dans le monde actuel du locuteur (ou du moins, ce qui est présenté comme tel par le locuteur), et ce qui est (ou pourrait être) le cas dans un autre monde possible. Avec cette distinction, importée de la sphère modale, je désire souligner que ce qui importe, ce n'est pas tellement le locuteur en tant que centre déictique, mais l'optique qu'il choisit, son point de vue sur la situation décrite." (1987 : 96) A l'intérieur de la division principale entre l'actuel et le non-actuel, Herslund opère une distinction secondaire entre emplois déictiques et emplois non-déictiques et obtient ainsi le classement suivant : (1987: 97)

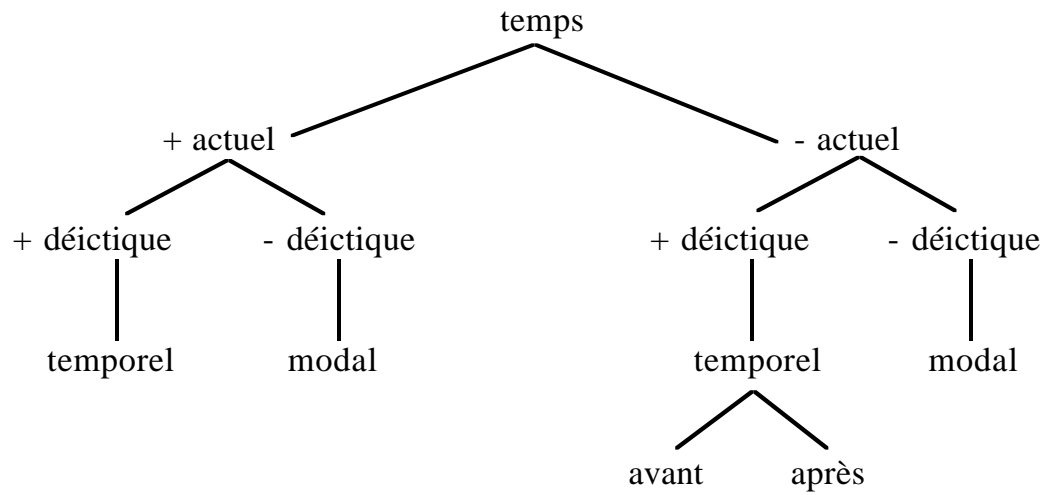

Les formes de présent peuvent désigner aussi bien l'actuel que le nonactuel du locuteur. Dans j'ai faim le présent désigne le monde actuel. Renvoyant à la situation d'énonciation, son emploi est déictique. Il désigne de même le monde actuel, mais avec une valeur modale lorsqu'il figure dans des phrases rapportées à toutes les époques : tous les hommes sont mortels... Qui va à la chasse perd sa place. Ce présent se présente comme vrai dans tous les temps, donc indépendamment de son moment de parole, et indépendamment de son locuteur. Il est le temps privilégié de l'énoncé de définition et de l'énoncé proverbial. Dans les phrases hypothétiques du type : si tu pars, je ne partirai pas la valeur du présent réside dans sa référence à un monde non-actuel. Contrairement au présent qui peut désigner aussi bien l'actuel que le non-actuel, les formes de passé et de futur désignent toujours le non-actuel : "dans leur 
emploi déictique, elles [les formes du passé] réfèrent au monde du passé (qui n'existe plus), dans leur emploi non-déictique, elles réfèrent à un monde imaginé (qui n'existe pas actuellement, mais qui pourrait exister ou, même, qui va exister)." (1987 : 98) Il en va de même pour les formes de futur dont le sens découle de leur valeur de non-actuel. Dans leur emploi déictique elles réfèrent à un monde qui va exister, dans leur emploi non-déictique, elles réfèrent à un monde imaginé.

Dans son étude traitant les formes temporelles dans les textes, Herslund rapproche son système alternatif modo-temporel de la distribution des formes verbales étudiée par $\mathrm{H}$. Weinrich, qui lui aussi nie la relation temporelle avec le moment de la parole. Weinrich procède à une classification des temps en deux groupes, laquelle se fonde sur deux attitudes de locution et qu'il appelle récit et commentaire : "Une façon de résoudre les problèmes posés par l'emploi des formes temporelles dans différents textes, dit Herslund, est d'admettre, avec Weinrich 1973(1964), que ces formes ne réfèrent pas principalement au Temps, mais qu'elles reflètent une attitude ou une intention : à l'aide des formes du présent, on commente le monde tel qu'il est, avec des formes de passé, on raconte un monde, on le crée." (1987 : 99)

Contrairement à Weinrich, pour qui la valeur et le sens des formes temporelles sont leur fonction textuelle, Herslund soutient que les formes temporelles ont les valeurs qui ressortent de sa conception du temps et acquièrent par là certaines fonctions textuelles : "Une des fonctions textuelles des formes du passé, celle qui consiste à raconter le monde, est dérivée de leur référence inhérente à un monde non-actuel. La fonction essentielle des formes du présent, en revanche, celle qui consiste à commenter le monde, est dérivée de leur référence au monde actuel du locuteur." (1987 : 99) "Dans cette optique, continue Herslund, le fait que le présent historique soit extrêmement fréquent, aussi bien dans des textes historiques que dans des textes de fiction, n'a rien d'étrange : la fonction textuelle du présent est justement de commenter, c'est-à- dire de signaler qu'il s'agit d'un reportage. L'historien, ou 'le narrateur' dans la fiction, commente le monde avec, comme centre déictique, sa propre personne." (1987 : 101)

Dans son étude du présent historique, le système alternatif que propose Herslund a, à première vue, l'avantage par rapport au système traditionnel déictique de n'avoir pas à considérer le présent historique comme une distorsion à effet stylistique, "une métaphore temporelle" 
selon Weinrich (1973 : 226) - explication insatisfaisante pour les linguistes - par laquelle le narrateur se reporte en arrière ou bien fait, en imagination, revivre les événements passés dans le présent de sa narration. Par cet emploi du présent historique, dit O. Jespersen en suivant la même tradition, "le locuteur oublie, si l'on peut dire, toute référence temporelle et se rappelle l'histoire qu'il rapporte avec autant de netteté que si elle était en train de se dérouler sous ses yeux." (1933:239)

D'autres linguistes interprètent cette "métaphore temporelle" par un" transport" du lecteur vers les événements : le lecteur est incité à accompagner le déroulement du procès comme s'il assistait à l'événement ou participait à la situation décrite.

Selon les Anciens, un effet d'hypotypose se produit. Quintilien définit ainsi cette figure : "Image des choses, si bien représentée par la parole que l'auditeur croit plutôt la voir que l'entendre." (Institution oratoire, IX, 2)

Comme nous venons de le voir, le "transport" évoqué par la "métaphore temporelle" implique soit l'événement soit le lecteur. La question de savoir si avec l'énoncé au présent historique nous le ramenons vers nous ou si nous sommes ramenés vers lui est formulée explicitement par A. Klum : "Est-ce que par le présent historique on transporte l'événement passé à l'actualité du sujet parlant ou s'agit-il par contre d'un report à l'époque où se déroulent les événements?" (1961 : 161) Les linguistes sont partagés à ce sujet et y répondent de deux manières distinctes. Tantôt c'est l'événement qui se détache du passé et se présente devant le locuteur: "Les faits, que l'on sait appartenir au passé, sont présentés comme s'ils appartenaient au présent le plus actuel." (P. Imbs, 1960 : 201) Tantôt, à l'inverse, c'est le locuteur qui "se transporte" dans le passé : "[Le présent] exprime des faits passés quand le locuteur se jette avec vivacité dans le récit." (J. Damourette et E. Pichon, 19111936, T. 5 : § 2086)

La nécessité de postuler un "saut" métaphorique, un "comme si", est liée à la fois à la définition de la valeur de base du présent comme renvoi à l'actuel et à l'identification souvent abusive d'un locuteur ou d'un narrateur à un énonciateur. La conception métaphorique du présent dans le récit a de même pour conséquence de lui attribuer comme allant de soi, un effet de "vivacité", qui est fréquemment démenti par les textes et, à juste titre, remis en cause par H. Sten (1952). Nous reviendrons plus loin sur cette discussion pour démontrer que si métaphore il y a, 
celle-ci peut s'expliquer par la valeur aspectuelle du présent et par l'approche du repérage temporel du présent historique.

Si le système modo-temporel de Herslund a le mérite de mettre en évidence les insuffisances de l'approche déictique du temps et si à première vue son approche permet de mieux rendre compte du présent historique, il nous semble pourtant que le présent historique n'est pas sans poser quelques problèmes dans le système alternatif que nous propose Herslund. Il signale avec raison que le présent ne réfère pas à quelque chose de simultané avec le moment de parole mais considère néanmoins que la valeur du présent historique est d'ordre déictique. Il explique les occurrences du présent en contexte narratif passé par le fait que "la fonction textuelle du présent est de commenter, c'est-à-dire de signaler qu'il s'agit d'un reportage. L'historien, ou le narrateur dans la fiction, commente le monde avec, comme centre déictique, sa propre personne." Or, le problème, c'est que le présent historique et le passé simple dans beaucoup de textes historiques, mémoires et textes de fiction alternent sans qu'on puisse associer cette alternance à une transition du récit au commentaire ou inversement. C'est d'ailleurs ce que doit reconnaître Herslund lui-même dans son étude du texte de François de Closets, lequel fait alterner des segments au passé simple et des segments au présent historique dans un souci de variation. Voici le texte :

"On s'est querellé à propos de l'inégalité française bien avant de la connaître.

Curieusement, ce n'est pas en France mais depuis l'étranger que le débat fut lancé. En 1969, la Commission économique de l'O.N.U. publia un document à sensation sur l'inégalité dans 13 pays. Les experts utilisaient comme indicateur l'écart entre les revenus des $10 \mathrm{p}$. 100 les plus riches et des 10 p. 100 les plus pauvres de la population. On atteignait 1 à 36 pour l'Inde, 1 à 25 pour le Brésil et, stupeur! 1 à 73 pour la France. La patrie de la "Liberté - Égalité - Fraternité" était la plus inégalitaire des nations. L'opposition s'empara de ces chiffres...l'I.N.S.E.E. aussi. Les staticiens français n'eurent aucune peine à démontrer que leurs collègues onusiens avaient comparé ce qui n'était pas comparable et que ces conclusions étaient "dénuées de tout fondement".

Nos chercheurs pouvaient dénoncer une erreur grossière, mais ils étaient incapables d'établir une vérité certaine. Cet épisode eut tout de même le mérite de les inciter à étudier de plus près la réalité monétaire française. Les premières recherches sérieuses commencèrent. 
Nouvelle "affaire" en 1976. Cette fois, c'est l'O.C.D.E. qui publie une comparaison internationale, établie par un économiste britannique, Malcolm Sawyer. 10 pays ont été étudiés selon 7 critères. Pour 4 d'entre eux, la France vient en tête des inégalités. Au total, elle se trouve classée après la Suède ou la Grande-Bretagne, ce qui n'est guère surprenant, mais également derrière le Canada, les États-Unis ou l'Italie. Deux fois plus inégale que l'Allemagne, trois fois plus que les Pays-Bas, voilà qui justifie les titres de la presse : "La France médaille d'or de l'inégalité", "La France première au palmarès international de l'inégalité", entre autres exemples."

(François de Closets : Toujours plus! p. 149)

Nous ne pouvons observer ni une dramatisation ni une tension particulière dans cette alternance. Ou si tension il y a, celle-ci est due à un brusque changement de temps, qui produit un effet de rupture dans le texte. C'est ce qu'a signalé $\mathrm{S}$. Mellet dans son étude du présent historique et son alternance avec le passé simple dans La Guerre des Gaules de César et dans les Mémoires de Guerre de de Gaulle (1980). Comme elle dit “il arrive que le présent historique n' ait aucune valeur de dramatisation ; il suffit pour cela qu'il soit le temps majoritaire du récit, donc du banal." (1980 : 10) De ses observations S. Mellet tire la conclusion suivante : C'est le changement de temps qui produit l'effet stylistique et le présent, pas plus qu'un autre, n'exprime par lui-même la dramatisation du récit." (1980: 11)

Quant à l'emploi du présent dans le texte de François de Closets, on peut se demander si nous pouvons parler de présent historique ou s'il n'est pas plutôt question d'un présent de résumé d'un rapport que le journaliste aurait sous les yeux et dont il résumerait le contenu essentiel par rapport au sujet de son article. Le présent dont il est ici question donne une vision globale des faits, c'est-à-dire que les faits sont non focalisés (focalisation zéro dans la terminologie de Genette).

Lorsque Herslund affirme que "en choisissant telle ou telle forme temporelle, on choisit une optique de commentaire ou une optique de récit" (1987 : 104) et qu'en choisissant le présent historique, le locuteur "choisit de mettre certaines choses en relief, de les actualiser" (1987 : 105), il est proche de l'affirmation de Weinrich selon laquelle "en employant les temps commentatifs [le présent, le futur et le passé composé], je fais savoir à mon interlocuteur que le texte mérite de sa part une attention vigilante [Gespannheit]. Par les temps du récit, au contraire, je 
l'avertis qu'une autre écoute plus détachée [Entspannheit] est possible." (1973: 30)

Cette opposition entre "tension" et "détente" ne nous paraît pas recouvrir l'opposition de deux stratégies discursives : il s'agit beaucoup plus d'une exigence de toute communication ; par ailleurs l'opposition commentaire/récit, tout comme l'opposition discours/histoire de Benveniste, repose sur une distribution des formes qui est loin d'être généralisée. Il est tout à fait hasardeux de construire une dichotomie d'attitudes sur des ensembles de formes exclusifs. Dans les textes de fiction comme dans les textes de non-fiction, la répartition des énoncés entre les deux plans obéit à des contraintes essentiellement textuelles. Elle ne découle pas directement du signifié des verbes. On peut difficilement prétendre qu'il y aurait des verbes que leur signifié vouerait irrémédiablement au premier ou à l'arrière-plan, au commentaire ou au récit. Citons comme exemple l'imparfait de rupture qui renvoie l'énoncé au premier et non pas à l'arrière-plan :

"Il avait rendez-vous dans la steppe avec Dieu lui-même, et il se $\underline{h a}-$ tait lorsqu'il rencontra un paysan dont la voiture était embourbée. Alors saint Dimitri l'aida.. La boue était épaisse, la fondrière profonde. Il fallut batailler pendant une heure. Et quand ce fut fini, saint Dimitri courut au rendez-vous. Mais Dieu n'était plus là."

(A. Camus, Les Justes, cité par Weinrich)

Il est clair que la situation exprimée par l'imparfait Mais Dieu n'était plus là, ne peut pas être mise au second plan. C'est autour d'elle que s'organise tout le récit, elle en donne une explication et une conclusion. Par ailleurs, certains passés simples sont au second plan ou relèvent manifestement d'un commentaire. Voici un exemple, cité par L. Waugh :

"On a souvent dit, à l'occasion des heures difficiles, et elles furent nombreuses dans la vie de la communauté [commentaire], que nous arrivons à un moment décisif..." (Le Monde : 1/5 1984)

Notons de même que dans les récits fictifs ou non-fictifs modernes entièrement au présent, la frontière entre narrer et commenter est effacée. Dans le récit au présent, on ne peut pas prétendre que le présent commente plus qu'il ne raconte. Pour reprendre les termes de Benveniste, on peut dire que l'on peut raconter aussi bien sur le mode de l'énonciation historique que sur celui de l'énonciation de discours.

Il n'en reste pas moins que les occurrences du présent en contexte narratif passé, dans beaucoup de textes, comme le deuxième texte de 
90

Frédéric Pottecher étudié par Herslund, semblent bien "actualiser" et "mettre en relief" certains éléments au présent historique dont l'emploi "correspond à une "intrusion évaluative" dans la narration des événements.” (1987:108) Voici le texte :

"En conséquence, Philippe Pétain demande à "Son Excellence le Chef de l'État grand-allemand" de lui donner la possibilité de rentrer en France, car, au surplus, écrit-il :

...Je veux défendre mon honneur et protéger par ma présence ceux qui m'ont servi. Aucun argument ne saurait me faire renoncer à ce projet.

A mon âge, je ne crains qu'une chose, c'est de n'avoir pas fait tout mon devoir. Je veux faire le mien jusqu'au bout.

La lecture de cette lettre provoqua un certain silence. Puis le bâtonnier Payen indiqua que la réponse de Hitler au Maréchal consista à faire enlever ce dernier (et sa suite) du château de Sigmaringen, et à diriger tout ce petit monde dans quelque point reculé du réduit bavarois. Mais le Maréchal s'y opposa et, après des aventures, l'officier qui commandait cette expédition abandonna le Maréchal et sa suite à la frontière suisse. Le Maréchal vint alors au poste-frontière de Vallorbe se livrer aux autorités françaises...

Le bâtonnier Payen affirme ensuite que son illustre client n'a jamais voulu supprimer la République. L'Assemblée nationale, le 10 juillet 1940, a donné "tous pouvoirs au gouvernement de la République sous l'autorité et la signature du maréchal Pétain". Donc, poursuit le bâtonnier Payen, en droit, la République n'a pas cessé d'exister. Or, on l'avait oublié, le président de la République, au terme de la Constitution de 1875, ne peut être mis en accusation que par la Chambre des députés et ne peut être jugé que par le Sénat. La Constitution de 1875 doit être appliquée; l'ordonnance du 13 novembre 1944, instituant la Haute Cour est nulle puisque la République n'a pas cessé d'exister."

(Frédéric Pottecher : Le procès Pétain, p. 30.)

Le texte de Pottecher met en scène plusieurs locuteurs. Nous assistons ainsi à deux scènes : la scène au discours direct sous la forme d'une lettre du maréchal Pétain qui remonte à l'année 1944. Ensuite apparaît la scène du procès en 1945 lors de laquelle nous assistons à l'argumentation du défenseur Payen sous la forme de discours indirect et de discours indirect libre. Dans ces deux scènes au présent, c'est la focalisation interne (Genette) qui domine. Les événements (faits) sont vus et racontés respectivement du point de vue de Pétain et du point de vue de Payen, ce qui fait que le lecteur assiste aux événements en direct et qu'il 
a l'impression, l'impact du vécu et de présence. Les autres parties qui portent sur les événements de l'été 1944 et les réactions suscitées par la lecture de la lettre lors du procès sont dominées par la focalisation zéro. Elles sont racontées au passé simple et les événements sont repérés les uns par rapport aux autres. C'est évidemment, comme l'indique Herslund, Pottecher qui choisit de montrer certains faits sous forme de scène et de narrer certains d'autres sous forme de récit, mettant ainsi les premiers en relief.

Le présent historique n'a pas - contrairement à ce que dit Herslund pour fonction d'actualiser au sens temporel du terme, mais bien au sens fictionnel. Les personnages-acteurs - qui sont ici Pétain et surtout Payen - sont montrés dans l'accomplissement de leur action, alors que dans un compte rendu de la réalité, le prétérit sert plutôt à marquer les actions accomplies, à les donner pour faits. Cette fonction de représentation dramatique ou directe du présent historique explique pourquoi on a l'impression que Pottecher et le lecteur ont la scène du procès de Pétain sous les yeux comme dans un drame.

\section{L'approche aspectuelle}

Nous retrouvons dans le domaine de l'aspect les mêmes divergences d'opinion constatées à propos des définitions temporelles. La conception la plus partagée définit le présent comme ayant la propriété aspectuelle d'imperfectif (R. Martin) ou d'inaccompli (P. Le Goffic).

Selon G. Serbat, il est vrai que dans tous les cas où il y a une absence de marqueurs détachant l'énoncé de la situation d'énonciation, le repérage s'effectue par rapport au moment de la parole, le procès est ouvert à droite et le présent renvoie effectivement à de l'inaccompli. Mais même lorsque le moment de référence demeure le présent de l'énonciation, il suffit que soit introduit un ordre de successivité entre une série de procès de type événement pour que la valeur d'inaccompli n'ait plus cours, toujours d'après Serbat, pour qui, dans le cas classique du présent de reportage, les actions de reportage sont 'accomplies, 'achevées', 'parfaites' au moment même où elles sont décrites. (1980 : 36). Serbat cite l'exemple suivant :

Dubois intercepte la balle, feinte le troisième ligne gallois Smith, mais l'arrière Baker surgit et l'accroche... Dubois passe à Dupont qui marque entre les poteaux." ( $1980: 36)$. 
Pour notre part, nous dirons que les actions sont énoncées au fur et à mesure de leur inscription dans le temps, leur enchaînement nous amenant à les percevoir chacune dans leur intégralité intérieure avant que ne s'énonce la suivante. C'est sans doute ce que Serbat entend par "achevé". Il n'en reste pas moins que les actions sont vues en cours d'accomplissement, ce qui explique que les actions du reportage sont vues et données à voir en direct - comme nous venons de le voir dans le texte de Pottecher. Pour un reportage sportif, l'art du commentateur consiste justement à nous faire vivre mentalement la scène dans son déroulement même, en saisissant ses principaux moments avec une attention aiguë, en les énonçant selon un rythme de parole adapté à la vitesse ou à la lenteur de leur survenance, et en utilisant le présent. Transposé au récit au passé simple, l'effet de sens d'une saisie "sur le vif" serait perdu.

L'effet de sens d'une saisie sur le vif est dû à la valeur aspectuelle de la forme du présent historique. Avec le présent historique, l'image du procès est construite à partir d'un repère interne et mobile fourni par le déroulement du procès lui-même. Cette valeur spécifique ressemble à l'image de ce que J.P. Desclés a appelé le "référentiel énonciatif" (1995 : 10) : image d'un intervalle borné à gauche et ouvert à droite pour laquelle le repère essentiel n'est pas le repère temporel externe (passé, futur, actuel ou indéterminé) mais bien le repère aspectuel interne, situé sur la borne droite ouverte du procès et accompagnant point par point l'inscription de celui-ci dans le réel. C'est ce que nous voyons dans l'exemple suivant, où nous suivons un voyage en cours d'accomplissement:

Comme j'étais contraint d'attendre le départ du bateau à vapeur pour le Caire [...] je voulus utiliser mon temps et me résolus à faire le court voyage de Rosette. [...]

A ma droite, dans le sud, s'aplatit une lande humide qui tient la place de l'ancien lac Maréotis; au fond s'allonge une ligne droite et brillante qui est le canal Mahmoudieh. Après avoir traversé un petit bois de tamarix inclinés sous le vent, nous entrons sur une grève où se voient encore des obus et des biscaïens demeurés là depuis les gloires de notre première république; le sable se continue jusqu'à une pointe de terre armée d'un fort qui baigne ses pieds dans la mer : c'est Aboukir!

Nous suivons la grève; le pied de nos chevaux enfonce dans le sable mouillé ; une sorte de masse noirâtre et couverte de coquillages s'élève au-dessus des eaux [...] 
Des marsouins sautent dans les vagues et $\underline{\text { montrent leur dos luisant. }}$

Nous sommes, avec le murmure affaibli de la mer, le seul bruit de la nature.

Le delta, que les Arabes nomment "rif", est, comme tu le sais, coupé à sa base par une multitude de lacs, dont quelques-uns sont fort importants ; j'arrivai auprès du lac d'Edkou, qui se dégorge dans la Méditerranée par un canal naturel où coulait une belle eau verdâtre; nous le traversâmes en bateau, et ce ne fut pas sans peine, car la mule qui portait mes bagages refusa longtemps et obstinément d'entrer dans la barque.

(Maxime Du Camp, Le Nil, Egypte et Nubie, 1854, Paris, Hachette, 1977, pp.16-17)

Comme il est évident que le narrateur n'a pas écrit en allant à cheval, le caractère historique du présent apparaît clairement, nous savons que le voyage relève nécessairement d'un passé, proche ou lointain. Il n'empêche que dans l'extrait en question, on suit le regard au fil d'un voyage, si bien que l'on a l'impression d'une reproduction quasi filmique non seulement des paysages, mais de chaque pas du voyage au moment même où il s'accomplit. C'est évidemment cette impression qui explique l'interprétation métaphorique selon laquelle le narrateur se déplace dans sa propre expérience passée, l'actualisant pour lui-même comme pour le lecteur. Selon nous, cette impression est due également à la saisie ouverte du procès qui évoque l'image d'un processus en cours, comparable au processus de l'énonciation qui le produit.

Il nous semble que l'approche aspectuelle du présent peut tout à fait être combinée avec l'approche de Culioli et celle de Vuillaume.

\section{L'approche du repérage temporel}

Puisque le présent historique alterne souvent avec le passé simple, certains linguistes ont appelé ce présent un "présent aoristique". Parler de "présent aoristique" n'est pas sans poser quelques problèmes, car cette forme n'est pas perçue comme l'équivalent d'un passé simple, lui aussi aoristique. Pour rendre compte de cette différence, Culioli (1980 : 185) a suggéré que le repère de ce présent ne soit ni lié à la situation d'énonciation (le moment de parole) comme le présent déictique ni coupé de celle-ci comme le passé simple, mais situé par rapport à un repère "fictif" construit à partir du moment d'énonciation : un repère qui serait à la fois coupé de ce moment d'énonciation et identifié à lui. 
Dans cette perspective, le présent historique est donc non pas repéré par rapport au moment de parole (à la situation d'énonciation) mais par rapport à un repère-origine fictif, c'est-à-dire que le moment du locuteur n'a pas de valeur propre et c'est le moment du lecteur, quel qu'il soit, qui actualise le moment du locuteur et lui donne une valeur. Cela explique la question impressionnante du "transport" du locuteur ou de l'événement. Par l'opération de décrochage aoristique, le locuteur construit, en rupture par rapport à lui-même, un co-énonciateur fictif ; et c'est par rapport à la nouvelle origine que constitue ce dernier que le récit au présent peut ensuite être repéré.

Le repère-origine fictif correspond au repérage de la fiction secondaire de Vuillaume. Ce dernier distingue la fiction fondamentale, qui correspond à ce qu'on appelle communément l'histoire ou le récit des événements, et la fiction secondaire, qui met en scène le narrateur et le lecteur et les présente comme des témoins oculaires des événements narrés. Vuillaume distingue deux niveaux de repérage qui correspondent à deux configurations de l'univers fictif : la configuration étendue et la configuration restreinte. Dans le cas de la configuration étendue, les expressions déictiques (et le présent) sont repérés par rapport à l'énonciation présumée du narrateur, et dans le cas de la configuration restreinte, l'actualité correspond à l'époque des événements que le lecteur est en train de lire et qui sont représentés devant ses yeux au moment de la lecture. L'univers est restreint en ce sens qu'il ne comprend que le niveau des événements : il n'y a pas repérage par rapport à l'énonciation. Puisque le présent historique ne figure que dans la configuration restreinte, nous allons nous limiter à ce cas.

Vuillaume limite son analyse à la fiction narrative, mais son cadre théorique s'applique aussi à certains récits historiques dans la mesure où l'historiographe-narrateur peut, lui aussi comme le narrateur fictif, créer un univers, faire une "représentation" de l'histoire. Traits caractéristiques de la fiction secondaire : d'une part les récits se présentent comme s'ajustant après coup à une réalité passée, c'est-à-dire antérieure à la date de leur production ; mais d'autre part, ils possèdent la propriété singulière de ressusciter l'univers qu'ils décrivent.

La question de savoir si c'est le narrateur qui reproduit les événements au présent ou si c'est le lecteur qui redescend dans le temps n'a pas beaucoup d'importance. En fait les deux perspectives reviennent au même et sont compatibles, comme le signale d'ailleurs Vuillaume lui- 
même : “Objectivement, les événements narrés appartiennent au passé, mais la fiction [secondaire] possède la propriété de les faire revivre dans le présent ou, du moins, de susciter le sentiment de cette résurrection. De ce point de vue, on peut dire que le passé (en fait son image) "remonte" vers le présent. Mais pour le lecteur, c'est l'inverse qui se produit : lui, en effet, a le sentiment de "descendre" dans le temps, jusqu'à devenir le contemporain des personnages du récit et partager leur récit". (1990 . 87) Dans cette perspective, le présent historique exprime la simultanéité par rapport au centre déictique qui est ici la fiction secondaire.

Voici un exemple de configuration restreinte :

Et maintenant il faut que nos lecteurs laissent voguer le Standard [...] et nous suivent au camp royaliste, situé en deça de la Tyne, auprès de la ville de Newcastle. C'est là, placées entre deux rivières [...] que s'étalent les tentes d'une petite armée[...]. A l'une des extrémités du camp[...], un homme vêtu en cavalier dort, couché sur le gazon et la main droite étendue sur son épée. A cinquante pas de là, un autre homme, vêtu aussi en cavalier, cause avec une sentinelle écossaise[...]. Comme une heure du matin sonnait à la ville de Newcastle, le dormeur s'éveilla.

( A. Dumas, Vingt ans après, Gallimard : Bibliothèque de la Pléiade, p. 1249. Exemple cité par Vuillaume).

Cette longue interruption au présent fixe des arrêts sur image, où se retrouve l'effet dominant du présent scénique. Le présent installe ici un poste d'observation pour le narrateur - dont le centre déictique est la fiction secondaire - un relais entre l'auteur et les événements, mais un relais mobile qui permet de suivre en direct le cours des événements. Simultanément, on voit la feinte : le décalage avec les autres faits, rapportés au passé, inscrit dans le tissu narratif une distance intérieure. Alors, ce qu'on perçoit, à côté d'une contemporanéité projetée par le narrateur, c'est le pouvoir du présent de générer indéfiniment une actualité dans la conscience du lecteur. Ce présent se déploie donc dans un univers à deux actualités : celle purement imaginaire du narrateur, et celle en puissance du lecteur.

Le texte de Pottecher est, de même, un exemple de configuration restreinte. Grâce à l'emploi du présent historique et à la mise en scène directe des protagonistes et des événements, le moment du procès est actualisé par chaque lecture qui transporte l'historiographe et le lecteur du lieu et du moment où ils se situent effectivement jusqu'à la salle 
d'audience et l'année 1945 où se déroule le procès de Pétain. L'art de Pottecher consiste à transporter le lecteur dans l'univers du procès où il assiste à la lecture de la lettre de Pétain et ensuite aux arguments du défenseur Payen pour ainsi donner à l'illusion du déplacement la plus grande force de persuasion. C'est bien la raison pour laquelle on peut dire que l'emploi du présent historique "correspond à une "intrusion évaluative" dans la narration des événements". Car cette évaluation est à la fois celle de Pottecher et celle du lecteur.

Le repérage temporel par rapport à la fiction secondaire fonctionne, de même, dans les ouvrages historiques. Voici des exemples de fiction secondaire du type configuration réstreinte trouvés chez Michelet:

"Traversons maintenant la rue Saint-Honoré, et voyons comment, presque en face, les meneurs de l'Assemblée, les royalistes constitutionnels, réunis aux Feuillants dans les locaux des comités, voyons comment ils emploient leur nuit."

(Histoire de la révolution française, vol. 1, p. 547)

"Venez voir, je voue prie, ce peuple couché par terre, pauvre Job, entre ses faux amis, ses patrons, ses fameux sauveurs, le clergé, la royauté. Voyez le douloureux regard qu'il lance au roi sans parler.” (Id., p. 86)

Les exemples ci-dessus montrent que le narrateur et le lecteur participent à l'univers des événements historiques. Ils traversent les rues, s'approchent pour mieux voir ce qui s'y passe et rencontrent les protagonistes de l'histoire. Le présent coïncide avec la date des événements narrés. Les marques de présent, en effet, prennent leur sens par rapport à la date des événements que le narrateur raconte et dont le lecteur est en train de prendre connaissance. Même si le narrateur invite son lecteur à assister à des événements qui se sont déroulés dans un passé largement antérieur au moment où il est en train de lire, la fiction secondaire créée fait ressusciter les événements de sorte que la narration et les faits rapportés semblent simultanés comme dans le cas d'un reportage.

\section{Conclusion}

L'approche aspectuelle et l'approche du repérage temporel du présent historique nous ont permis de rendre compte de la fonction de représentation dramatique et directe de ce présent. Cette technique de représentation propre au récit de fiction s'est répandue dans certaines formes de 
récits de non-fiction, telles l'autobiographie, le récit de voyage et le récit historique.

Les narratologues font depuis longtemps la distinction entre l'auteur qui imagine et invente l'histoire et le narrateur qui non seulement raconte l'histoire mais qui en fait aussi partie en tant que témoin plus ou moins “visible". Comme l'historien n'est pas l'auteur de l'histoire qu'il relate, il n'est pas libre d'inventer les incidents ou les personnages qui formeront le corps de son récit. Or, comme il n'existe pas de fiction pure, il n'existe pas d'Histoire si rigoureuse qu'elle s'abstienne de toute "mise en intrigue" et de tout procédé romanesque. L'historien est donc tout à fait libre d'épouser le point de vue de tel ou tel et de se mettre à la place de tel ou tel. C'est ce que nous voyons dans le texte de Pottecher, dans lequel l'historien épouse respectivement le point de vue de Pétain et celui de Payen et leur cède la parole, se limitant à les mettre en scène. En employant le présent historique, Pottecher ne commente pas le monde, comme le dit Herslund, mais il nous le "montre". L'auteur du récit de voyage peut "se déplacer" dans sa propre expérience passée, l'"actualisant" pour lui-même comme pour le lecteur. C'est ce qui se produit lorsqu'il épouse le point de vue de son moi-antérieur (ex. le récit de Maxime Du Camp). Dans l'histoire romancée de Michelet, l'auteur se donne un pouvoir que normalement l'historien n'a pas : il participe en tant que narrateur-témoin aux événements historiques. $\mathrm{Ce}$ faisant, il a recours à des procédés de "fictionalisation" comme le présent historique, ce qui explique que le présent n'a pas pour fonction d'actualiser au sens temporel - mais bien au sens fictionnel.

Culioli a démontré que le présent historique n'est pas repéré par rapport au moment de parole mais par rapport à un repère-origine fictif qui correspond à la fiction secondaire de Vuillaume. Comme ce repère est à la fois coupé du moment de parole et identifié à lui, il invite à faire la distinction entre le locuteur ou le narrateur et l'énonciateur, distinction qui correspond de près à celle entre le narrateur et l'auteur. Grâce à ce repérage temporel du présent historique, nous n'avons pas à postuler un "saut" métaphorique - mais il contribue néanmoins à l'expliquer tout en mettant en évidence le relais qu'installe le présent entre l'auteur et le narrateur - relais mobile qui permet de suivre en direct le cours des événements. 


\section{Références bibliographiques}

Benveniste, E. (1966, 1974). Problèmes de linguistique générale. Paris : Gallimard, Tomes I et II.

Comrie, B. (1976). Aspect. Cambridge : Cambridge University Press.

Comrie, B. (1985). Tense. Cambridge : Cambridge University Press.

Culioli, A. (1980). Valeurs aspectuelles et opérations énonciatives : l'aoristique. In David, J. Et Martin, R. (Eds.). La Notion d'aspect. Actes du Colloque organisé par le Centre d'Analyse syntaxique de l'Université de Metz. Paris : Klincksieck, pp. 181- 193.

Damourette, J. et Pichon, E. (1911-1936). Des mots à la pensée. Essai de grammaire de la langue française, Tome 5. Paris : Edition J.1. D’Artrey.

Desclés, J.P. (1995). Les référentiels temporels pour le temps linguistique. In Modèles linguistiques XVI 2, pp.9-36.

Genette, G. (1972). Figures III. Paris : Editions du Seuil.

Genette, G. (1983). Nouveau discours du récit. Paris : Editions du Seuil.

Herslund, M. (1987). Catégories grammaticales et linguistique textuelle : la catégorie du temps en français. In Copenhagen Studies in Language no. 10, pp. 89-108.

Imbs, P. (1960). L'Emploi des temps verbaux en français moderne : essai de grammaire descriptive. Paris : Klincksieck.

Jespersen, O. (1933). Essentials of English Grammar. Londres : G. Allen and Unwin.

Klum, A. (1961). Verbe et adverbe. Uppsala/Stockholm : Almquist \& Wiksell.

Le Goffic, P. (1986). Que l'imparfait n'est pas un temps du passé. In Points de vue sur l'imparfait. Caen : Centre de Publications de Caen, pp. 55-68.

Lyons, J.C. (1977). Semantics I-II. Cambridge : Cambridge University Press.

Martin, R. (1971). Temps et aspect. Paris : Klincksieck.

Mellet, S. (1980). Le présent 'historique' ou 'de narration'. In L'information grammaticale no. 4, pp. 6-11.

Serbat, G. (1980). La place du présent de l'indicatif dans le système des temps. In L'information grammaticale no. 7, pp. 36-39.

Sten, H. (1952). Les temps du verbe fini (indicatif) en français moderne. Copenhague : Munksgaard.

Vuillaume, M. (1990). Grammaire temporelle des récits. Paris : Editions de Minuit.

Vuillaume, M. (1993). Le repérage temporel dans les textes narratifs. In Langages 112, pp. 92-105.

Waugh, L. et Monville-Burston, M. (1986). Aspect and Discourse function. In Language 62, pp. 846-877.

Weinrich, H. (1973). Le temps. Paris : Editions du Seuil. Edition originale : Tempus, Stuttgart, 1964.

Wilmet, M. (1976). Etudes de morpho-syntaxe verbale. Paris : Klincksieck. 